\title{
An Ovariectomy-Induced Rabbit Osteoporotic Model: A New Perspective
}

\author{
Nathan Robert Wanderman ${ }^{1}$, Cindy Mallet ${ }^{2}$, Hugo Giambini ${ }^{2}$, Nirong Bao ${ }^{2}$, \\ Chunfeng Zhao ${ }^{2}$, Kai-Nan An ${ }^{2}$, Brett A. Freedman ${ }^{1}$, Ahmad Nassr ${ }^{1}$ \\ ${ }^{1}$ Department of Orthopedic Surgery, Mayo Clinic, Rochester, MN, USA \\ ${ }^{2}$ Biomechanics Laboratory, Division of Orthopedic Research, Rochester, MN, USA
}

\section{Study Design: Experimental Animal Model.}

Purpose: The aim of our study was to validate a pure bilateral ovariectomy (OVX) female New Zealand white rabbit model of postmenopausal osteoporosis utilizing animal-sparing in vivo techniques for evaluating bone mineral density (BMD). We also sought to demonstrate that bilateral OVX in female New Zealand white rabbits can produce diminished BMD in the spinal column and simulate osteoporosis, without the need for adjuvant chemotherapeutic agents (i.e., no additional glucocorticosteroids or other drugs were used for stimulating accelerated BMD loss), which can be assessed by in vivo BMD testing.

Overview of Literature: Multiple animal models of postmenopausal osteoporosis have been described. Rat ovariectomy models have been successful, but are limited by rats' inability to achieve true skeletal maturity and a slight morphology that limits surgical instrumentation. Rabbit models have been described which do not have these limitations, but previous models have relied on adjunctive steroid therapy to achieve osteoporosis and have required animal sacrifice for bone mineral density assessment.

Methods: Thirty-six skeletally mature female rabbits underwent bilateral OVX. BMD was measured using dual-energy X-ray absorptiometry on the metaphysis of the proximal tibia and distal femur, at baseline and 17 weeks postoperatively.

Results: Mean BMD values were significantly reduced by $21.9 \%(p<0.05)$ in the proximal tibia and $11.9 \%(p<0.001)$ in the distal femur at 17 weeks.

Conclusions: This study is the first to demonstrate a significant bone loss within four months of pure OVX in rabbits using animalsparing validation techniques. We believe that this OVX model is safe, reproducible, and can be employed to longitudinally evaluate the effect of anti-osteoporosis therapeutics and surgical interventions.

Keywords: Osteoporosis; Animal; Ovariectomy; Bone remodeling

\section{Introduction}

Primary osteoporosis, including postmenopausal and ageassociated osteoporosis, is a global public health concern. Osteoporotic animal models are widely used for testing the efficacy and the effect of therapeutic and pharmaceutical drugs. Multiple animal models, mostly using rodents, have been used for mimicking postmenopausal osteoporosis [1,2]. Rats are commonly utilized because of their low price and ease in handling and housing. It has been

Received Mar 8, 2017; Revised May 4, 2017; Accepted May 22, 2017

Corresponding author: Brett A. Freedman

Department of Orthopedic Surgery, Mayo Clinic, 200 First Street SW, Rochester, MN 55905, USA

Tel: +1-507-266-5262, Fax: +1-507-266-2533, E-mail: freedman.brett@mayo.edu 
demonstrated that ovariectomy (OVX) in rats can induce cancellous bone deficiency-simulating postmenopausal bone loss $[1,2]$. However, the rodent model has several disadvantages including inability in achieving true skeletal maturity, lack of a Haversian system with minimal intra-cortical bone remodeling, and a slight morphometry that prohibits surgical experimentation of implant fixation and construct rigidity $[1,2]$. Conversely, rabbits have a short developmental period, larger anatomic elements, and reach true sexual maturity between 20 and 24 weeks with bone maturity and closure of growth plates between 28 and 32 weeks $[3,4]$. They also show active Haversian remodeling analogous to that observed in humans and demonstrate faster bone turnover than rodents and primates [4-13]. Lastly, they are a commonly used animal model for spine surgery research and they have a sufficient structural size to allow for performance of common surgical interventions (i.e., instrumented fusion) for assessing the impact of induced osteoporosis on these procedures [14,15].

Multiple osteoporotic rabbit models have been described, including reducing dietary calcium, glucocorticoid administration, and bilateral OVX; many combining several techniques for achieving faster rates of osteoporosis [16-22]. However, relatively few publications describe rabbit models with OVX as a singular mechanism for modeling osteoporosis, and of those publications, few utilize the trabecular bone for measuring bone mineral density (BMD) [11-13,23-25]. In this context, an animalsparing rabbit model of postmenopausal osteoporosis validated using an in vivo $\mathrm{BMD}$ analysis of the trabecular bone within 4 months of OVX is under-developed as a technique or under-reported in the literature.

The aim of our study was to validate a pure OVX rabbit model of postmenopausal osteoporosis that utilized animal-sparing techniques for evaluating BMD. We hypothesized that OVX alone, without additional therapeutic interventions, could induce postmenopausal osteoporosis in rabbits as measured by trabecular BMD. Current literature suggests that osteoporosis, in rabbits, cannot be reliably induced by OVX alone [17]. To overcome this perceived limitation, others have used adjuvants like glucocorticoids to hasten and increase the magnitude of BMD loss following OVX; however, these strategies introduce mechanisms that more closely mimic secondary osteoporosis. This study is unique for its attempt in creating and validating a model of significant bone loss in rabbits following bilat- eral OVX alone, reproducing the mechanisms of primary osteoporosis. We intend to demonstrate that this osteoporotic rabbit model is safe, reproducible, and can be used to further understand the action of bone remodeling agents in postmenopausal osteoporosis without the interaction of glucocorticoids or other model-specific adjuvants.

\section{Materials and Methods}

Thirty-six skeletally mature, 6-month-old, white New Zealand female rabbits were used for this study. Pre-OVX, the mean \pm standard deviation weight of the rabbits was $3.6 \pm 0.3 \mathrm{~kg}$. All animals were kept in the same environment and conditions. The rabbits were housed in individual cages and were fed ad libitum with water and standard commercial rabbit food. They were maintained on a 12 hour light and 12-hour night cycle at room temperature $\left(20^{\circ} \mathrm{C}\right)$ with $58 \%$ humidity. Animals were acclimatized in those conditions for at least 5 days before the start of experiments. Animal care and experimental procedures were approved by our Institutional Animal Care and Use
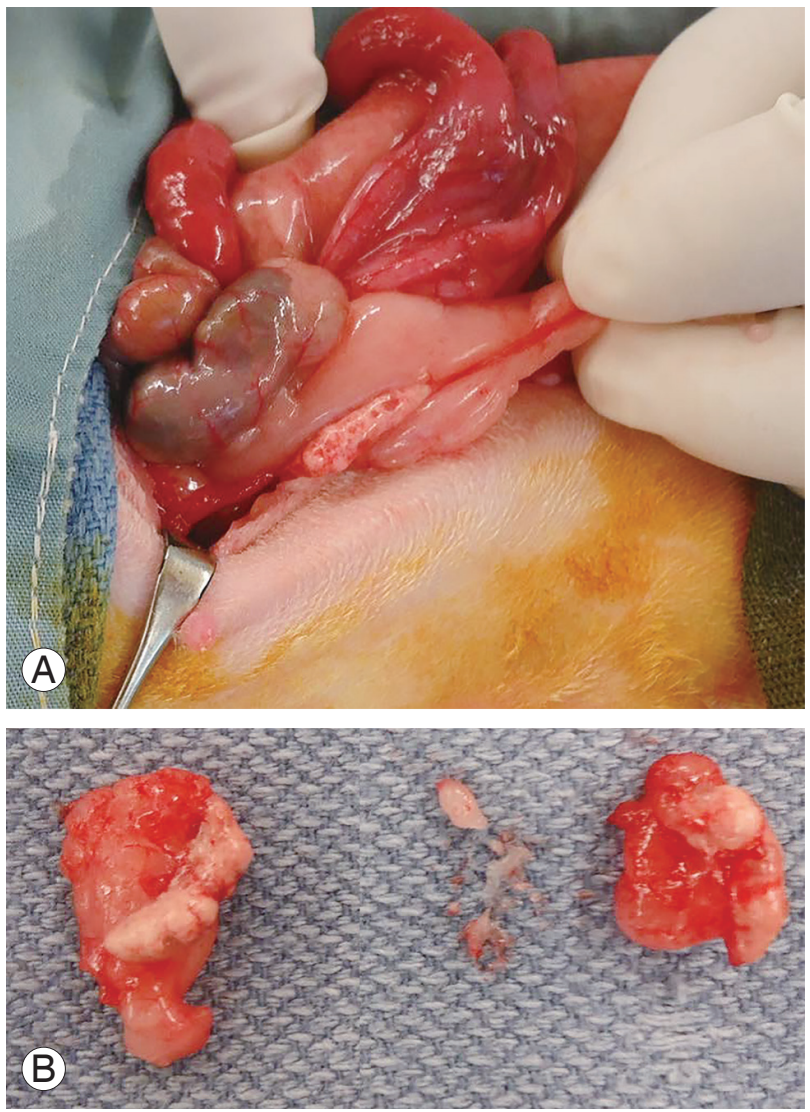

Fig. 1. (A) Ovariectomy procedure and (B) excised ovaries during surgical intervention. 
Committee (approval no., A39712-12; The chair of the Institutional Animal Care Use Committee is Dr. Hirohito Kita.).

\section{Bilateral ovariectomy}

Under general anesthesia, the rabbits were injected with Buprenorphine SR $0.15 \mathrm{mg} / \mathrm{kg}$, intubated and maintained using Isoflurane $1.5 \%-3 \%$ with oxygen. All rabbits underwent bilateral OVX. A midline incision was distally made from the umbilicus for $4-5 \mathrm{~cm}$. The linea alba and peritoneal tissue below were incised, thereby protecting the intestines. The ovaries were then localized, and the ovarian vessels were ligated. The ovaries were removed with their ligamentous attachment on the uterine horn (Fig. 1). The linea alba and abdominal muscles were then closed followed by the skin using an absorbable suture (Vicryl 3.0; ETHICON Inc., Somerville, NJ, USA). Animals were then observed twice a day until healing of the incision had occurred.

\section{Bone mineral density measurements}

BMD measurements were acquired using a dual-energy X-ray absorptiometry (DXA) system (Lunar Piximus 2; GE-Lunar, Madison, WI, USA). The system was calibrated daily, and the Lunar Piximus 2 ver. 2.0 software (GELunar) was used to measure BMD, bone area, and bone mineral content. The rabbits were placed under general anesthesia consisting of an intramuscular injection of ketamine $(40 \mathrm{mg} / \mathrm{kg})$ and xylazine $(6 \mathrm{mg} / \mathrm{kg})$. Measurements were performed in vivo at the level of the right proximal tibia and distal femur (Fig. 2). The region of interest (ROI) was the center of the proximal tibia and distal femur metaphysis, located in the bone $1 \mathrm{~cm}$ below and $2 \mathrm{~cm}$ above the joint line, respectively. The mean chosen ROI was 0.25 $\mathrm{cm}^{2}$ for the proximal tibia and $0.10 \mathrm{~cm}^{2}$ for the distal femur. BMD was measured on the femur and tibia for each rabbit at baseline (time of OVX) and 17 weeks after OVX.

\section{Statistical analysis}

The bone mass was measured at baseline and 17 weeks within ROI of the tibia and femur. Mean BMD at baseline and post OVX were compared using univariate analysis performed with a paired Student $t$-test. Significance was set at $p$-value of $<0.05$.

\section{Results}

Thirty-three rabbits survived the study while three rabbits died ( $8 \%$ attrition) because of respiratory failure due to induction of anesthesia at the time of the OVX. No postoperative complications were observed. Wound healing occurred at approximately 5 days. Preoperatively, the mean rabbit weight was $3.6 \pm 0.3 \mathrm{~kg}$, increasing to $4.5 \pm 0.3$ $\mathrm{kg}$ at 17 weeks post OVX. Five femora were unavailable for measurement because of difficulty in placement of
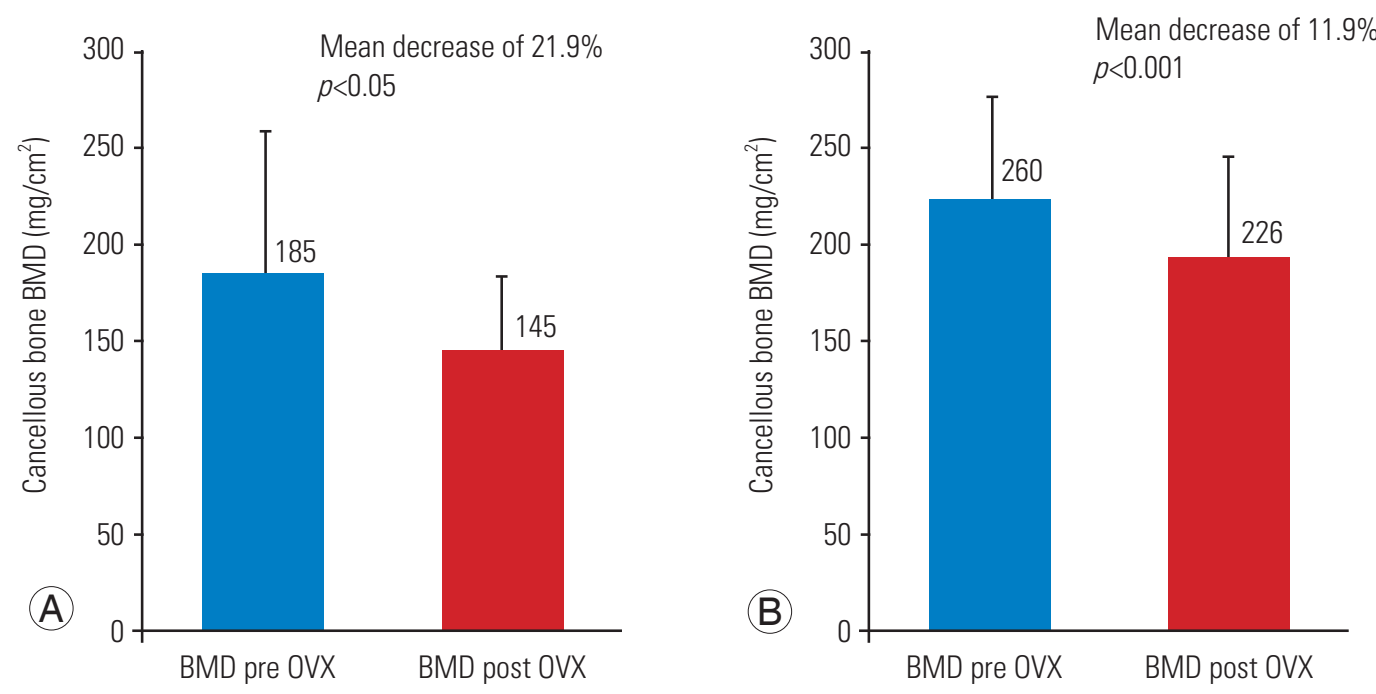

Fig. 2. Trabecular BMD measured at the proximal tibia (A) and distal femur (B) of rabbits (in $\left.\mathrm{mg} / \mathrm{cm}^{2}\right)$. Measurements were performed in vivo before the surgical procedure and 17 weeks post ovariectomy. BMD, bone mineral density; OVX, ovariectomy. 
Table 1. Summary of mean BMD measurements for the tibia and femur at baseline and 17 weeks after the OVX procedure

\begin{tabular}{lccc} 
& & Cancellous bone BMD $\left(\mathrm{mg} / \mathrm{cm}^{2}\right)$ & \\
\cline { 2 - 4 } Variable & Baseline & 17 Weeks after OVX & Change \\
\cline { 2 - 4 } & $185.1 \pm 72.4$ & $144.5 \pm 38.1$ & $-40.6^{*}$ \\
Proximal tibia metaphysis $(\mathrm{n}=33)$ & $259.6 \pm 60.6$ & $225.9 \pm 59.9$ & $-33.7^{* *}$ \\
\hline
\end{tabular}

Values are presented as mean \pm standard deviation.

$\mathrm{BMD}$, bone mineral density; OVX, ovariectomy; n, sample number.

${ }^{*} p<0.05 .{ }^{* *} p<0.001$.

the rabbits during the acquisition of the images, while proximal tibial measurements were achieved in all surviving animals. The mean BMD value of the proximal tibia and distal femur metaphysis at the time of surgery and 17 weeks post OVX are shown in Table 1 and expressed in $\mathrm{mg} / \mathrm{cm}^{2}$. Cancellous BMD of the tibia and femur decreased from 185.1 to $144.5 \mathrm{mg} / \mathrm{cm}^{2}$ and 259.6 to 225.9 $\mathrm{mg} / \mathrm{cm}^{2}$, respectively. During a 17 -week period post OVX, mean BMD values of the proximal tibia and distal metaphysis significantly decreased by $40.6 \mathrm{mg} / \mathrm{cm}^{2}$ or $21.9 \%$ $(p<0.05)$ and $33.7 \mathrm{mg} / \mathrm{cm}^{2}$ or $11.9 \%(p=0.001)$, respectively (Fig. 2).

\section{Discussion}

Estrogen deprivation by bilateral OVX alone has been previously described as a pure model of postmenopausal osteoporosis in rabbits [11-13,23-25]. However, previous studies have been limited by the period from OVX to BMD validation, BMD measurement using nontrabecular bones, or reliance on animal sacrifice for BMD validation. The aim of this study was to establish a model of primary osteoporosis which mitigates those limitations and that can be used for assessing the efficacy of antiosteoporosis therapeutics and surgical adjuvants.

Our results show a significant loss of BMD in the proximal tibia (21.9\%) and distal femur (11.9\%) in rabbits 17 weeks post bilateral OVX, as measured by DXA of the proximal tibia and distal femur. These preliminary results support our hypothesis that OVX in rabbits induces significant bone loss in the trabecular bone within 18 weeks. Furthermore, this bone loss was measurable via DXA, which enabled animal-sparing BMD validation. Ultimately, we believe that our OVX monotherapy model of postmenopausal osteoporosis can assist in future research of chemotherapeutic and surgical interventions for osteoporosis.
Multiple studies have validated a rabbit osteoporosis model utilizing OVX along with glucocorticoid supplements (daily intramuscular injection of methylprednisolone sodium succinate, $1 \mathrm{mg} / \mathrm{kg} /$ day for $4-8$ weeks, depending on the study), as previous studies failed to show early BMD reduction with OVX monotherapy $[3,16,24,26]$. For instance, Baofeng et al. [16] found no significant difference in L3-L4 BMD at 6 and 10 weeks post OVX compared with baseline values $(6.5 \%$ and $8.5 \%$ decrease, respectively). Castaneda et al. [17] demonstrated a trend toward BMD reduction in the lumbar spine and global knee but no significant reduction at 6 and 16 weeks post OVX. These studies compared a small number of rabbits, ranging from 4 to 7 , which may not have been sufficient to reach a significant difference.

Other studies involving OVX monotherapy have shown a significant BMD reduction if given a longer time course, but this prolonged period limits the usefulness of these models for in vivo research on the impact of osteoporosis on spine surgery. Ye et al. [12] studied 20 rabbits utilizing an OVX model which confirmed femoral BMD reduction, but only at 8 months post OVX, presenting a lengthy time course for practical use as an animal osteoporosis model. Yildiz et al. [13] described an OVX model involving 12 rabbits achieving tibial BMD reduction in the course of 16 weeks. However, their validation technique utilized animal sacrifice for measurement of BMD, which diminishes the versatility of the model for testing interventions that may require longitudinal BMD monitoring. Pennypacker et al. [11] described an OVX model that validated trabecular bone loss in 13 weeks, but also relied on animal sacrifice for BMD measurement. Arslan et al. [23] described reduced callus formation using 12 OVX rabbits and 12 control rabbits subjected to tibia metaphyseal distraction via Ilizarov external fixators, but lacked evaluation of BMD in existing bone. Sevil et al. [25] described an OVX model, and did confirm BMD reduction at 16 weeks fol- 
lowing OVX, but only using ex vivo techniques.

Prior rabbit models of osteoporosis using OVX monotherapy have failed to validate trabecular BMD reduction using an animal-sparing in vivo technique within a 4-month timeframe. Our results support a direct relationship between ovarian function loss and trabecular bone loss in rabbits within 17 weeks using animal-spearing DXA scanning as validation. Our findings show that the OVX osteoporotic rabbit model is safe, reproducible, and can be used to longitudinally study the action of bone remodeling agents and surgical interventions in postmenopausal osteoporosis without the interaction of glucocorticoids or other model-specific adjuvants.

The current study has several limitations. First, we quantified bone loss in the tibia and femur using DXA. Although, clinically used as a gold standard for osteoporosis diagnosis and fracture risk prediction, additional measurements, such as micro-computed tomography, histology, and bone turnover biomarkers could have further verified BMD measurements and bone architecture. Secondly, though previous studies have indicated that rabbits subjected to a sham OVX do not experience loss in femoral or tibial BMD [12], our study did not contain a sham control group for evaluation of aging-related bone loss. Thirdly, we evaluated BMD only at the time of OVX and at the 17-week mark. Additional scans would have been useful for characterizing the rate of BMD reduction. Finally, due to our animal-sparing approach, we found it to be impractical to perform live DXA scans of rabbit vertebrae, and had to rely on only femur and tibia BMD measurements for validation. Future studies should utilize a larger number of rabbits to support a large sham control group, and perform additional imaging and BMD analysis.

\section{Conclusions}

We were able to demonstrate significant bone loss in a rabbit model induced by bilateral OVX alone. We observed a decrease of $21.9 \%$ and $11.9 \%$ in BMD of the proximal tibia and femur, respectively, 17 weeks post OVX. This was validated using an animal-sparing DXA scan. Our OVX rabbit model of postmenopausal osteoporosis appears to be safe and reproducible and induces bone loss within four months. We believe that this OVX model can be employed to longitudinally evaluate the effect of anti-osteoporosis therapeutics and surgical inter- ventions.

\section{Conflict of Interest}

No authors have any conflict of interest.

\section{Acknowledgments}

This project was supported by internal funding from AO Spine North America.

\section{References}

1. Kalu DN. The ovariectomized rat model of postmenopausal bone loss. Bone Miner 1991;15:175-91.

2. Turner AS. Animal models of osteoporosis: necessity and limitations. Eur Cell Mater 2001;1:66-81.

3. Castaneda S, Calvo E, Largo R, et al. Characterization of a new experimental model of osteoporosis in rabbits. J Bone Miner Metab 2008;26:53-9.

4. Gilsanz V, Roe TF, Gibbens DT, et al. Effect of sex steroids on peak bone density of growing rabbits. Am J Physiol 1988;255(4 Pt 1):E416-21.

5. Chen X, Giambini H, Ben-Abraham E, An KN, Nassr A, Zhao C. Effect of bone mineral density on rotator cuff tear: an osteoporotic rabbit model. PLoS One 2015;10:e0139384.

6. Dai L, Wu H, Yu S, et al. Effects of OsteoKing on osteoporotic rabbits. Mol Med Rep 2015;12:1066-74.

7. Jensen PR, Andersen TL, Pennypacker BL, Duong LT, Delaisse JM. The bone resorption inhibitors odanacatib and alendronate affect post-osteoclastic events differently in ovariectomized rabbits. Calcif Tissue Int 2014;94:212-22.

8. Li JP, Li P, Hu J, et al. Early healing of hydroxyapatite-coated implants in grafted bone of zoledronic acid-treated osteoporotic rabbits. J Periodontol 2014;85:308-16.

9. Lozano D, Trejo CG, Gomez-Barrena E, et al. Osteostatin-loaded onto mesoporous ceramics improves the early phase of bone regeneration in a rabbit osteopenia model. Acta Biomater 2012;8:2317-23.

10. Oki Y, Doi K, Makihara Y, Kubo T, Oue H, Tsuga K. Intermittent administration of parathyroid hormone enhances primary stability of dental implants in a bone-reduced rabbit model. J Oral Sci 2016;58:241- 
6.

11. Pennypacker BL, Duong LT, Cusick TE, et al. Cathepsin $\mathrm{K}$ inhibitors prevent bone loss in estrogendeficient rabbits. J Bone Miner Res 2011;26:252-62.

12. Ye X, Zhang P, Xue S, Xu Y, Tan J, Liu G. Adiposederived stem cells alleviate osteoporosis by enhancing osteogenesis and inhibiting adipogenesis in a rabbit model. Cytotherapy 2014;16:1643-55.

13. Yildiz A, Esen E, Kurkcu M, Damlar I, Daglioglu K, Akova T. Effect of zoledronic acid on osseointegration of titanium implants: an experimental study in an ovariectomized rabbit model. J Oral Maxillofac Surg 2010;68:515-23.

14. Boden SD, Schimandle JH, Hutton WC. An experimental lumbar intertransverse process spinal fusion model: radiographic, histologic, and biomechanical healing characteristics. Spine (Phila Pa 1976) 1995;20:412-20.

15. Hu Y, Leung HB, Lu WW, Luk KD. Histologic and electrophysiological changes of the paraspinal muscle after spinal fusion: an experimental study. Spine (Phila Pa 1976) 2008;33:1418-22.

16. Baofeng L, Zhi Y, Bei C, Guolin M, Qingshui Y, Jian L. Characterization of a rabbit osteoporosis model induced by ovariectomy and glucocorticoid. Acta Orthop 2010;81:396-401.

17. Castaneda S, Largo R, Calvo E, et al. Bone mineral measurements of subchondral and trabecular bone in healthy and osteoporotic rabbits. Skeletal Radiol 2006;35:34-41.

18. Li GW, Tang GY, Liu Y, Tang RB, Peng YF, Li W. MR spectroscopy and micro-CT in evaluation of osteoporosis model in rabbits: comparison with histopathology. Eur Radiol 2012;22:923-9.
19. Martin-Monge E, Tresguerres IF, Blanco L, Khraisat A, Rodriguez-Torres R, Tresguerres JA. Validation of an osteoporotic animal model for dental implant analyses: an in vivo densitometric study in rabbits. Int J Oral Maxillofac Implants 2011;26:725-30.

20. Oue H, Doi K, Oki Y, et al. Influence of implant surface topography on primary stability in a standardized osteoporosis rabbit model study. J Funct Biomater 2015;6:143-52.

21. Wen B, Zhu F, Li Z, Zhang P, Lin X, Dard M. The osseointegration behavior of titanium-zirconium implants in ovariectomized rabbits. Clin Oral Implants Res 2014;25:819-25.

22. Zhang J, Chen SG, Habaerxi K, et al. Reinforcing effect of calcium sulfate cement bovine bone morphogenetic protein on vertebral in the rabbit model of osteoporosis. Asian Pac J Trop Med 2014;7:3825.

23. Arslan H, Ketani A, Gezici A, et al. The effects of osteoporosis on distraction osteogenesis: an experimental study in an ovariectomised rabbit model. Acta Orthop Belg 2003;69:67-73.

24. Liu X, Lei W, Wu Z, et al. Effects of glucocorticoid on BMD, micro-architecture and biomechanics of cancellous and cortical bone mass in OVX rabbits. Med Eng Phys 2012;34:2-8.

25. Sevil F, Kara ME. The effects of ovariectomy on bone mineral density, geometrical, and biomechanical characteristics in the rabbit femur. Vet Comp Orthop Traumatol 2010;23:31-6.

26. Kaveh K, Ibrahim R, AbuBakar MZ, Ibrahim TA. Osteoporosis induction in animal model. Am J Anim Vet Sci 2010;5:139-45. 